\title{
The multiplicator of a regular product of groups
}

\section{William Haebich}

It is shown that if $G$ is an arbitrary regular product of its subgroups $A_{\lambda}, \lambda \in I$, then the multiplicator, $M(G)$, of $G$ is the direct product of the $M\left(A_{\lambda}\right)$ together with a certain other group. This extends a calculation of $M\left(A_{1} \times A_{2}\right)$ due to Schur. As an application, we find the multiplicator of a verbal wreath product $A \mathrm{wr}_{V} B$ where $A$ is abelian. A representing group for a finite regular product is also constructed.

\section{Regular products}

In this section we define regular products and list some of their properties needed in Section 2. Properties (1.7) and (1.8) are of particular importance. Everything here can be found in Golovin [2].

DEFINITION 1.1. $G$ is a regular product of its subgroups $A_{\lambda}$, $\lambda \in I$, where $I$ is an ordered set, if they generate $G$ and ${ }_{\lambda} \cap \hat{A}_{\lambda}=E$ where $\hat{A}_{\lambda}=\operatorname{sgp}\left(A_{\mu}^{G} \mid \mu \in I, \mu \neq \lambda\right)$. The $A_{\lambda}$ are called regular factors of $G$.

As usual $A_{\mu}^{G}$ denotes the normal closure of $A_{\mu}$ in $G$ and $E$ is the trivial group. From now on in this note we consider $G$ to be a fixed group generated by its subgroups $A_{\lambda}, \lambda \in I$. The statement " $G$ is a regular product of its subgroups $A_{\lambda}$ " will be abbreviated to " $G$ is a Received 17 May 1972. Communicated by P.J. Cossey. 
regular product".

If $X_{\lambda}$ is an arbitrary subgroup of $G(\lambda \in I)$ then the notation $\left[X_{\lambda}\right]$ will be used for the subgroup, $\operatorname{sgp}\left(\left[x_{\lambda}, x_{\mu}\right] \mid x_{\lambda} \in X_{\lambda} ; x_{\mu} \in X_{\mu} ; \lambda, \mu \in I ; \lambda \neq \mu\right)$, called the cartesion of the $X_{\lambda}$.

The product $\prod_{\lambda \in I} x_{\lambda}$ will mean the set, in $G$, of all elements of the form $x_{\lambda_{1}} x_{\lambda_{2}} \cdots x_{\lambda_{n}}$ where $\lambda_{j} \in I$ and $\lambda_{1}<\lambda_{2}<\ldots<\lambda_{n}$. If each $x_{\lambda}$ is normal in $G$ then $\prod_{\lambda \in I} X_{\lambda}$ becomes the normal subgroup of $G$ generated by the $X_{\lambda}$ and any two orderings of $I$ will yield the same product.

(1.2). $\left[A_{\lambda}^{G}\right]$ is normal in $G$.

(1.3). If $G$ is generated by its subgroups $A_{\lambda}$ then $G=\left(\prod_{\lambda \in I} A_{\lambda}\right)\left[{ }_{A}^{G}\right]$. Furthermore $G$ is a regular product if and only if each of its elements can be written uniquely as a product $a_{\lambda_{1}} a_{\lambda_{2}} \cdots a_{\lambda_{n}}{ }^{u}$ where $a_{\lambda_{i}} \in A_{\lambda_{i}}, \quad \lambda_{1}<\lambda_{2}<\ldots<\lambda_{n}$ cond $u \in[A G]$.

(1.4). If $G$ is a regular product and $X_{\lambda}$ is a subgroup of $A_{\lambda}$ for each $\lambda \in I$, then the subgroup of $G$ generated by the $X_{\lambda}$ is a regular product of the $X_{\lambda}$.

(1.5). If $G$ is a regular product then $G^{\prime}=\left(\prod_{\lambda \in I} A_{\lambda}^{\prime}\right)\left[A_{\lambda}^{G}\right]$.

DEFINITION 1.6. If $G$ is a reguzar product, a homomorphism $\phi$ of $G$ to a group $\bar{G}$ is a regular homomorphism if ker $\phi$ is a subgroup of $[A, G]$ : hence the terms regular homomorphic image and regular quotient group.

(1.7). If $G$ is a regular product and $\phi: G \rightarrow \bar{G}$ is a regular 
homomorphism then $\phi$ restricted to $A_{\lambda}$ is on isomorphism for each $\lambda \in I$ and $G \phi$ is a regular product of the $A_{\lambda} \phi$.

(1.8). If $\psi: \prod_{\lambda \in I}^{*} A_{\lambda} \rightarrow G$ (where $\prod_{\lambda \in I}^{*} A_{\lambda}$ denotes the free product of the $A_{\lambda}$ ) is the natural homomorphism induced by the identity map on each $A_{\lambda}$, then $G$ is a.regular product if and only if $\psi$ is regular.

This last result shows that the free product of a set of groups can be interpreted as the largest regular product of those groups. In the same way, their direct product is the smallest such product. For, if $G$ is a regular product of the $A_{\lambda}$, then the quotient $G /\left[A_{\lambda}^{G}\right]$ is a direct product of copies of the $A_{\lambda}$.

\section{Calculation of the multiplicator}

We define the multiplicator of $G$ to be $R \cap F^{\prime} /[R, F]$ where $F / R$ is a presentation for $G$. The most direct way of approaching $M(G)$, when $G$ is a regular product, is to try to express $R \cap F^{\prime} /[R, F]$ in terms of the groups $R \cap F_{\lambda}^{\prime} /\left[\left(R \cap F_{\lambda}\right), F_{\lambda}\right]$ where $F_{\lambda}$ is the group of elements in $F$ which map onto $A_{\lambda} \cdot\left(F_{\lambda} / R \cap F_{\lambda}\right.$ is a presentation for $\left.A_{\lambda} \cdot\right)$ This is the sort of method previously applied to $M\left(A_{1} \times A_{2}\right)$ (see [8]). The difficulty here lies in not knowing how the $F_{\lambda}$ generate $F$. It can be avoided by taking a presentation, $F_{\lambda} / R_{\lambda}$, for each $A_{\lambda}$ and constructing a presentation for $G$ from the free product $\prod_{\lambda \in I}^{*} F_{\lambda}$.

In fact we begin with greater generality and let $B_{\lambda}$ be a fixed group which maps epimorphically onto $A_{\lambda}$ under $\nu_{\lambda}$. This will allow us to construct a representing group for $G$ when it is finite.

Suppose that $C_{\lambda}$ is the kernel of $v_{\lambda}$ and that $v$ is the natural epimorphism from the free product $B=\prod_{\lambda \in I}^{*}{ }^{B} \lambda$ onto $A=\prod_{\lambda \in I}^{*} A_{\lambda}$ induced 
by the $v_{\lambda}$. Further, if $\psi$ is the natural homomorphism from $A$ onto $G$ induced by the identity on each $A_{\lambda}$, let $H$ be the kernel of $\psi$ and $H_{0}$ the group in $B$ which maps onto $H$ under $v$. We have that

$$
\begin{aligned}
& H=H \cap\left[A_{\lambda}^{A}\right] \quad \text { since } \quad H \leq\left[A_{\lambda}^{A}\right] \quad \text { by (1.8) } \\
& =H_{0} \nu \cap\left[B_{\lambda}^{B}\right] \nu \text { since }\left[{ }_{B}^{B}\right]^{B} \nu=\left[B_{\lambda} \nu^{B} \nu\right] \text { by definition of the cartesian } \\
& =\left(H_{0} \cap\left[B_{\lambda}^{B}\right]\right) \nu \quad \text { since } \quad \text { ker } \nu \leq H_{0} \quad \text { by definition } \\
& =K \nu \quad \text { where } K=H_{0} \cap\left[B_{\lambda}^{B}\right] \text { is normal in } B \text {. }
\end{aligned}
$$

LEMMA 2.1. $B / C$ is isomorphic to $G$ where $c=\left(\prod_{\lambda \in I} c_{\lambda}^{B}\right) \cdot K$.

Proof. (i) We first show that kerv $=\prod_{\lambda \in I} c_{\lambda}^{B}$. (This is almost

certainly well known but I cannot find it in the literature.) The kernel of $\nu$ contains $\prod_{\lambda \in I} c_{\lambda}^{B}$ since, by construction of $\nu, C_{\lambda} \nu$ is trivial for each $\lambda \in I$. On the other hand,

$$
\begin{aligned}
B_{\mu} \cap \prod_{\lambda \in I} c_{\lambda}^{B} & =B_{\mu} \cap D_{\mu} C_{\mu}^{B} \quad \text { where } D_{\mu}=\prod_{\substack{\lambda \in I \\
\lambda \neq \mu}} c_{\lambda}^{B} \\
& =\left(B_{\mu} \cap D_{\mu}\left[C_{\mu}, B\right]\right) C_{\mu} \text { since } C_{\mu} \leq B_{\mu} \\
& =C_{\mu}
\end{aligned}
$$

by Definition 1.1 and the fact that $B_{\mu} \cap D_{\mu}\left[C_{\mu}, B\right] \leq B_{\mu} \cap \hat{B}_{\mu}$. Thus, if $\rho$ is the canonical homomorphism from $B$ onto $B / \prod_{\lambda \in I} c_{\lambda}^{B}$, then $B_{\lambda} \rho$ is isomorphic to $B_{\lambda} / C_{\lambda}$, and hence to $A_{\lambda}$.

Now $B \rho$ is generated by the $B_{\lambda} \rho, \lambda \in I$. Let $n$ be the natural epimorphism from $A$ onto $B_{\rho}$ induced by the isomorphism from each ${ }_{\lambda}$ to ${ }_{\lambda} \rho$. The following diagram commutes 


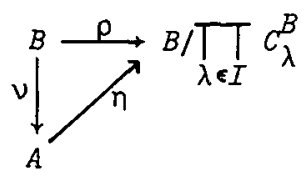

The kernel of $v$ is therefore contained in $\operatorname{ker} \rho=\prod_{\lambda \in I} c_{\lambda}^{B}$ and hence $\operatorname{kerv}=\prod_{\lambda \in I} c_{\lambda}^{B}$

(ii) The product, $\nu \psi$, of mappings is an epimorphism from $B$ onto G. Now,

$$
\begin{aligned}
& b \in \operatorname{ker} \nu \psi \Rightarrow b \nu \psi=1 \text { in } G \\
& \Leftrightarrow b \nu \in H \quad \text { since } H=\operatorname{ker} \psi \\
& \Longleftrightarrow b \in \operatorname{kerv} . K \text { since } K \nu=H \text {. }
\end{aligned}
$$

But

$$
\begin{aligned}
\text { kerv. } K & =\left(\prod_{\lambda \in I} c_{\lambda}^{B}\right) K \text { from }(i) \\
& =C .
\end{aligned}
$$

Thus $C=\operatorname{kerv} \psi$ from (i) and (ii).

We aim to find the structure of $C \cap B^{\prime} /[C ; B]$ which is isomorphic to $M(G)$ when $B$ is free by Lemma 2.1.

LEMMA 2.2. $\prod_{\lambda \in I} c_{\lambda}^{B}=\left(\prod_{\lambda \in I} c_{\lambda}\right) \cdot D$ in $B$ where $D=\prod_{\substack{\lambda, \mu \in I \\ \lambda \neq \mu}}\left[c_{\lambda}, B_{\mu}\right]^{B}$.

Proof. We prove that $c_{\lambda}^{b}$ is in $C_{\lambda} D$ for all $c_{\lambda} \in c_{\lambda}$ and $b \in B$ by induction on the length of the normal form for $b$. This is trivial when $b$ is of length one, for $c_{\lambda}^{b^{\mu}}=c_{\lambda}\left[c, b_{\mu}\right], \lambda \neq \mu$, and $c_{\lambda}^{b} \epsilon c_{\lambda}$ since $C_{\lambda}$ is normal in $B$. Suppose that $c_{\lambda}^{b} \in C_{\lambda} D$ for all elements $b$ of length $m$ and that $b b_{\mu}$ is a reduced word in $B$. Then 


$$
\begin{aligned}
c_{\lambda}^{b b_{\mu}} & =\left(c_{\lambda}^{\prime} d\right)^{b_{\mu}} \text { where } c_{\lambda}^{\prime} \in c_{\lambda}, d \in D \text { by the inductive hypothes is } \\
& =c_{\lambda}^{\prime}\left[c_{\lambda}^{\prime}, b_{\mu}\right] d^{b_{\mu}} \in c_{\lambda} D \text { since }\left[c_{\lambda}^{\prime}, b_{\mu}\right] \in D \text { for } \lambda \neq \mu,
\end{aligned}
$$

and if $\lambda=\mu$, then $\left[c_{\lambda}^{\prime}, b_{\lambda}\right] \in C_{\lambda}$.

Thus $C_{\lambda}^{B}$ is a subgroup of $C_{\lambda} D$. It follows that $\prod_{\lambda \in I} c_{\lambda}^{B}$ is a subgroup of $\prod_{\lambda \in I}\left(C_{\lambda} D\right)$. By definition, every element of $\prod_{\lambda \in I}\left(C_{\lambda} D\right)$ can be written in the form $c_{\lambda_{1}} d_{\lambda_{1}} c_{\lambda_{2}} d_{\lambda_{2}} \cdots c_{\lambda_{n}} d_{\lambda_{n}}$ where $c_{\lambda_{i}} \in c_{\lambda_{i}}$, $a_{\lambda_{i}} \in D$ and $\lambda_{1}<\lambda_{2}<\ldots<\lambda_{n}$. But $c_{\lambda_{1}} d_{\lambda_{1}} c_{\lambda_{2}} d_{\lambda_{2}} \doteqdot c_{\lambda_{n}} d_{\lambda_{n}}$

$$
=\left(c_{\lambda_{1}} c_{\lambda_{2}} \cdots c_{\lambda_{n}}\right) d_{\lambda_{1}}{ }^{\left(c_{\lambda_{2}} c_{\lambda_{3}} \cdots c_{\lambda_{n}}\right)}{ }_{d_{\lambda_{2}}}\left(c_{\lambda_{3}} \cdots c_{\lambda_{n}}\right) \ldots d_{\lambda_{n}}
$$

which belongs to $\left(\prod_{\lambda \in I} c_{\lambda}\right) D$. Hence $\prod_{\lambda \in I} c_{\lambda}^{B}$ is a subgroup of $\left(\prod_{\lambda \in I} C_{\lambda}\right) \cdot D$. The reverse inclusion is trivial since $\left[C_{\lambda}, B_{\mu}\right]^{B}$ is a subgroup of $C_{\lambda}^{B}$.

$$
\begin{aligned}
& \text { (2.3). (i) } C \cap B^{\prime}=\left(\prod_{\lambda \in I}\left(C_{\lambda} \cap B_{\lambda}^{\prime}\right)\right) \cdot D . K, \\
& \text { (ii) }[C, B]=\left(\prod_{\lambda \in I}\left[C_{\lambda}, B_{\lambda}\right]\right) D .[K, B] .
\end{aligned}
$$

Proof. (i) $C$ consists of all elements of the form $c=c_{\lambda_{1}} c_{\lambda_{2}} \cdots c_{\lambda_{m}} d k$ where $c_{\lambda_{i}} \in c_{\lambda_{i}}, d \in D, k \in K$, by the preceding two lemmas. The fact that $D K$ is in $B^{\prime}$ means that $c$ belongs to $B^{\prime}$ if and only if $\bar{c}=c_{\lambda_{1}} c_{\lambda_{2}} \cdots c_{\lambda_{m}}$ belongs to $B^{\prime}$. Now, by (1.5), every element of $B^{\prime}$ is of the form $b_{\lambda_{1}}^{\prime} b_{\lambda_{2}}^{\prime} \ldots b_{\lambda_{n}}^{\prime} u$ where 
The multiplicator of a product

285

$b_{\lambda}^{\prime} \in B_{\lambda}^{\prime}, u \in\left[B_{\lambda}^{B}\right]$. But both $C_{\lambda_{i}}$ and $B_{\lambda_{i}}^{\prime}$ are subgroups of $B_{\lambda_{i}}$. Therefore $\bar{c}$ is in $B^{\prime}$ if and only if $c_{\lambda_{i}}$ is in $B_{\lambda_{i}}^{\prime}$ by (1.2).

(ii)

(*)

$$
\begin{aligned}
{[C, B] } & =\left[\left(\prod_{\lambda \in I} c_{\lambda}^{B}\right) K, B\right] \\
& =\left(\prod_{\lambda \in I}\left[c_{\lambda}^{B}, B\right]\right)[K, B] \text { by }[2], \text { Lemma } 4.3 \\
& =\left(\prod_{\lambda \in I}\left[C_{\lambda}, B\right]^{B}\right)[K, B] .
\end{aligned}
$$

Now $\left[c_{\lambda}, B\right]$ is generated by the elements $\left[c_{\lambda}, b\right]$ where $c_{\lambda} \in c_{\lambda}$, $b \in B$. We can prove that $\left[c_{\lambda}, b\right] \in\left[C_{\lambda}, B_{\lambda}\right] D$ by induction on the length of $b$ precisely as in Lemma 2.2. The result is trivial for $b=b_{\mu}$.

Suppose that $\left[c_{\lambda}, b\right] \in\left[C_{\lambda}, B_{\lambda}\right] . D$ for $b$ of length $m$, then

$$
\begin{aligned}
& {\left[c_{\lambda}, b\right]=x_{\lambda} d \text { where } x_{\lambda} \in\left[c_{\lambda}, B_{\lambda}\right] \text { and } d \in D \text {. If } b b_{\mu} \text { is reduced then } } \\
&\left.\qquad c_{\lambda}, b b_{\mu}\right]=\left[c_{\lambda}, b_{\mu}\right]\left[c_{\lambda}, b\right]^{b} \\
&=\left[c_{\lambda}, b_{\mu}\right] x_{\lambda}{ }^{b_{\mu}} b_{\mu} \\
&=\left[c_{\lambda}, b_{\mu}\right] x_{\lambda}\left[x_{\lambda}, b_{\mu}\right] d^{b_{\mu}}
\end{aligned}
$$

which is an element of $\left[C_{\lambda}, B_{\lambda}\right] \cdot\left[C_{\lambda}, B_{\mu}\right] \cdot D$. Thus $\left[C_{\lambda}, B\right]$ is a subgroup of $\left[C_{\lambda}, B_{\lambda}\right] D$. Consequently,

$$
\begin{aligned}
{\left[C_{\lambda}, B\right]^{B} } & \leq\left[C_{\lambda}, B_{\lambda}\right]^{B} \cdot D^{B} \\
& =\left[C_{\lambda}, B_{\lambda}\right]\left[\left[C_{\lambda}, B_{\lambda}\right], B\right] D \\
& \leq\left[C_{\lambda}, B_{\lambda}\right]\left[C_{\lambda}, B\right] D \text { since } C_{\lambda} \leq B_{\lambda} \\
& \leq\left[C_{\lambda}, B_{\lambda}\right] D .
\end{aligned}
$$

Hence, from (*), $[C, B]$ is a subgroup of $\left(\prod_{\lambda \in I}\left(\left[C_{\lambda}, B_{\lambda}\right] D\right)\right) \cdot[K, B]$.

https://doi.org/10.1017/S0004972700045020 Published online by Cambridge University Press 
Again, copying the argument of Lemma 2.2, every element of $\prod_{\lambda \in I}\left(\left[C_{\lambda}, B_{\lambda}\right] D\right)$ is a product of the form $x_{\lambda_{1}} d_{\lambda_{1}} x_{\lambda_{2}} d_{\lambda_{2}} \cdots x_{\lambda_{n}} d_{\lambda_{n}}$ where $x_{\lambda_{i}} \in\left[c_{\lambda_{i}}, B_{\lambda_{i}}\right], d_{\lambda_{i}} \in D$ and $\lambda_{1}<\lambda_{2}<\ldots<\lambda_{n}$. This product can be rearranged to show that it lies in $\left(\prod_{\lambda \in I}\left[C_{\lambda}, B_{\lambda}\right]\right) D$. That is, $[C, B]$ is a subgroup of $\left(\prod_{\lambda \in I}\left[C_{\lambda}, B_{\lambda}\right]\right) D .[K, B]$. The reverse inclusion is trivial.

To shorten the proof of the main theorem, a technical lemma is established.

LEMMA 2.4. $\left[C_{\lambda},\left[B_{\mu}^{B}\right]\right]$ is a subgroup of $D$ for all $\lambda \in I$.

Proof. It suffices to prove that $\left[C_{\lambda}^{B},\left[B_{\mu}^{B}\right]\right]$ is a subgroup of $D$ since $\left[C_{\lambda},\left[B_{\mu}^{B}\right]\right]$ is a subgroup of $\left[C_{\lambda}^{B},\left[\begin{array}{l}B \\ B_{\mu}\end{array}\right]\right]$. The cartesian $\left[B_{\mu}^{B}\right]$ is generated by the elements $\left[b_{\mu}, b_{\rho}\right]$ where $b_{\mu} \in B_{\mu}^{B} ; b_{\rho} \in B_{\rho}^{B}$; $\mu, \rho \in I ; \mu \neq \rho$. Thus, because $c_{\lambda}^{B}$ is normal in $B,\left[C_{\lambda}^{B},\left[B_{\mu}^{B}\right]\right]$ is generated by the elements $\left[b_{\mu}, b_{\rho}, c_{\lambda}\right], b_{\mu} \in B_{\mu}^{B} ; b_{\rho} \in B_{\rho}^{B} ; c_{\lambda} \in c_{\lambda}^{B}$; $\mu \neq \rho$. Moreover,

$$
\begin{aligned}
{\left[C_{\lambda}^{B}, B_{\mu}^{B}\right] } & \leq\left[C_{\lambda} D, B_{\mu}^{B}\right] & \text { from Lemma } 2.2 \\
& =\left[C_{\lambda}, B_{\mu}^{B}\right]\left[D, B_{\mu}^{B}\right] & \\
& \leq D & \text { provided } \lambda \neq \mu .
\end{aligned}
$$

The lemma is therefore proved if $\left[b_{\mu}, b_{\rho}, c_{\lambda}\right], \mu \neq \rho$, can be shown to lie in $\left[C_{\lambda}^{B}, B_{\sigma}^{B}\right]$ for some $\sigma \neq \lambda$.

The following identity comes from Hall [3], (10.2.1.3), page 150,

(**) $\left[b_{\mu}, b_{\rho}, c_{\lambda}\right]=\left[c_{\lambda}, b_{\mu}^{-1}, b_{\rho}^{-1}\right]^{-b_{\mu} b^{-1}}\left[b_{\rho}^{-1}, c_{\lambda}^{-1}, b_{\mu}^{-1}\right]^{-c_{\lambda}^{-1} b_{1}^{-1}}$ 
Consider the first commutator. There are two cases. If $\lambda=\mu$ then $\left[c_{\lambda}, b_{\mu}^{-1}, b_{\rho}^{-1}\right]$ lies in $\left[C_{\lambda}^{B}, B_{\rho}^{B}\right]$ since $C_{\lambda}$ is normal in $B_{\lambda}(\lambda \neq \rho$ since $\mu \neq \rho$ by hypothesis). If $\lambda \neq \mu$ then

$$
\begin{aligned}
{\left[c_{\lambda}, b_{\mu}^{-1}, b_{\rho}^{-1}\right] } & =\left[c_{\lambda}, b_{\mu}^{-1}\right]^{-1}\left[c_{\lambda}, b_{\mu}^{-1}\right]^{b_{\rho}^{-1}} \\
& =\left[c_{\lambda}, b_{\mu}^{-1}\right]^{-1}\left[c_{\lambda}^{b_{\rho}^{-1}}, b_{\mu}^{-b_{\rho}^{-1}}\right],
\end{aligned}
$$

which is in $\left[c_{\lambda}^{B}, B_{\mu}^{B}\right]$. That is $\left[c_{\lambda}, b_{\mu}^{-1}, b_{\rho}^{-1}\right]^{-b_{\mu} b_{\rho}^{-1}}$ is in the normal subgroup $\left[C_{\lambda}^{B}, B_{\mathrm{p}}^{B}\right]$. The second commutator

$\left[b_{\rho}^{-1}, c_{\lambda}^{-1}, b_{\mu}\right]=\left[\left[c_{\lambda}^{-1}, b_{\rho}^{-1}\right]^{-1}, b_{\mu}\right]$ can be treated in the same fashion.

The result follows from (**).

THEOREM 2.5. $C \cap B^{\prime} /[C, B]$ is isomorphic to the direct product $\left(\prod_{\lambda \in I}^{\times} C_{\lambda} \cap B^{\prime} /\left[C_{\lambda}, B_{\lambda}\right]\right) \times H /[H, A]$.

Proof. The argument is split into three parts.

(i) Let $\phi$ be the canonical homomorphism from $B$ onto $B / D$. Then $\phi$ is a regular homomorphism since $\operatorname{ker} \phi=D$ and $D$ is clearly a subgroup of $\left[\begin{array}{l}B \\ B_{\lambda}\end{array}\right]$. It follows from (1.7) that $\phi$ restricted to $B_{\lambda}$ is an isomorphism for each $\lambda \in I$ and that $B \phi$ is a regular product of the ${ }^{B} \lambda^{\phi}$.

Consider $N=\operatorname{sgp}\left(C_{\lambda} \phi \mid \lambda \in I\right) . K \phi$ in $B \phi$. Now $\left[C_{\lambda}, C_{\mu}\right], \lambda \neq \mu$, is a subgroup of $D=\operatorname{ker} \phi$, thus

(a) $\left[C_{\lambda} \phi, C_{\mu} \phi\right]=E$ in $B \phi$.

Also 


$$
\begin{aligned}
& {\left[C_{\lambda}, K\right] \leq\left[C_{\lambda},\left[B_{\lambda}^{B}\right]\right] \text { since } K \leq\left[B_{\lambda}^{B}\right] \text { by construction }} \\
& \leq D \quad \text { by Lemme } 2.4 \text {. }
\end{aligned}
$$

Thus

(b)

$$
\left[C_{\lambda} \phi, K \phi\right]=E
$$

In addition,

$$
C_{\lambda} \phi \cap\left(\prod_{\substack{\mu \in I \\ \mu \neq \lambda}} c_{\mu} \phi\right) \cdot K \phi \leq B_{\lambda} \phi \cap\left(B_{\lambda} \phi\right)=E
$$

and

$$
\begin{aligned}
K \phi \cap \prod_{\lambda \in I} C_{\lambda} \phi & \leq\left[{ }_{\mu} \phi^{B \phi}\right] \cap \prod_{\lambda \in I} C_{\lambda} \phi \\
& =E \text { by }(1.3) .
\end{aligned}
$$

Conditions (a) to (d) are sufficient to make $N$ a direct product of its subgroups $K \phi$ and $C_{\lambda} \phi$.

$$
\begin{aligned}
C \cap B^{\prime} /[C, B] & \simeq \frac{C \cap B^{\prime} / D}{[C, B] / D} \text { since } D \leq[C, B] \text { from (2.3) (ii) } \\
& =\left(C \cap B^{\prime}\right) \phi /[C, B] \phi .
\end{aligned}
$$

From (i) and (2.3), we have that $\left(C \cap B^{\prime}\right) \phi$ is the direct product of its subgroups $K \phi$ and $\left(C_{\lambda} \cap B_{\lambda}^{\prime}\right) \phi, \lambda \in I$, (since $D \phi=E$ ) and that $[C, B] \phi$ is the direct product of its subgroups $[K, B] \phi$ and $\left[C_{\lambda}, B_{\lambda}\right] \phi, \lambda \in I$. This, together with the facts that $[K, B] \phi \leq K \phi$ and $\left[C_{\lambda}, B_{\lambda}\right] \phi \leq\left(C_{\lambda} \cap B_{\lambda}^{\prime}\right) \phi$, implies that

$$
\begin{aligned}
\left(C \cap B^{\prime}\right) \phi /[C, B] \phi & \simeq\left(\prod_{\lambda \in I}^{\times}\left(C_{\lambda} \cap B_{\lambda}^{\prime}\right) \phi /\left[C_{\lambda}, B_{\lambda}\right] \phi\right) \times K \phi /[K, B] \phi \\
& \simeq\left(\prod_{\lambda \in I}^{\times}\left(C_{\lambda} \cap B_{\lambda}^{\prime}\right) /\left[C_{\lambda}, B_{\lambda}\right]\right) \times K \phi /[K, B] \phi,
\end{aligned}
$$

since $\phi$ is an isomorphism on $B_{\lambda}$.

(iii) In conclusion, we prove that $K \phi /[K, B] \phi$ is isomorphic to 
$H /[H, A]$. Now,

$$
\begin{aligned}
K \phi /[K, B] \phi & \simeq \frac{K D / D}{[K, B] D / D} \quad \text { since } D=\operatorname{ker} \phi \\
& \simeq K D /[K, B] D \\
& =K[K, B] D /[K, B] D \text { since } K \leq B \\
& \simeq K / K \cap[K, B] \cdot D .
\end{aligned}
$$

Similarly

$$
K v /[K, B] \nu \simeq K / K \cap \operatorname{kerv} \cdot[K, B]
$$

But $K \cap \operatorname{kerv}[K, B]=K \cap\left(\prod_{\lambda \in I} c_{\lambda}^{B}\right)[K, B]$ since kerv $=\prod_{\lambda \in I} c_{\lambda}^{B}$ from Lemma

2.1 (i). Thus

$$
\begin{aligned}
K \cap \operatorname{kerv} \cdot[K, B] & =K \cap\left(\prod_{\lambda \in I} c_{\lambda}\right) D \cdot[K, B] \\
& =K \cap D[K, B] \quad \vdots \quad \text { since } K, D[K, B] \leq\left[\begin{array}{c}
B \\
B_{\lambda}^{B}
\end{array}\right] .
\end{aligned}
$$

Hence

$$
\begin{aligned}
K \phi /[K, B] \phi & \simeq K \nu /[K, B] \nu & \text { from (1) and (2) } \\
& =H /[H, A] & \text { since } K \nu=H \text { by construction. }
\end{aligned}
$$

The main result is a corollary to Theorem 2.5 .

THEOREM 2.6. $M(G)$ is isomorphic to the direct product $\left(\prod_{\lambda \in I}^{\times} M\left(A_{\lambda}\right)\right\} \times H /[H, A]$ when $G$ is a regular product of the $A_{\lambda}$ and $A / H \simeq G$ where $A=\prod_{\lambda \in I}^{*} A_{\lambda}$.

Proof. If $B_{\lambda}$ is a free group then $B_{\lambda} / C_{\lambda}$ is a presentation for $A_{\lambda}$ and $C_{\lambda} \cap B^{\prime} /\left[C_{\lambda}, B_{\lambda}\right]$ equals $M\left(A_{\lambda}\right)$. The free product, $B$, will be a free group and, since $B / C$ is isomorphic to $G, M(G)$ equals $C \cap B^{\prime} /[C, B]$. The result follows immediately from Theorem 2.5 .

Theorem 2.6 reduces the problem of finding $M(G)$ to that of finding $H /[H, A]$. When $G=A_{1} \times A_{2}, A=A_{1} * A_{2}$ and $H /[H, A]=\left[A_{1}, A_{2}\right] /\left[\left[A_{1}, A_{2}\right], A\right]$. Wiegold ([9], Lemma 3.9) shows that 
the latter group is isomorphic to $A_{1} \otimes A_{2}$. So we have the well known result, first proved by Schur, that $M\left(A_{1} \times A_{2}\right)$ is isomorphic to $M\left(A_{1}\right) \times M\left(A_{2}\right) \times\left(A_{1} \otimes A_{2}\right)$.

\section{Construction of a representing group}

There is a useful characterisation of $M(G)$, when $G$ is finite, due to Schur [7]. Of all the pairs of groups $(L, M)$ such that

$$
\begin{aligned}
& \text { (i) } L \text { is finite, } \\
& \text { (ii) } M \leq L^{\prime} \cap Z(L), \\
& \text { (iii) } L / M \simeq G,
\end{aligned}
$$

there exists an $L$ of maximal order called a representing group for $G$. The $M$ corresponding to such an $L$ is isomorphic to $M(G)$. We will call the pair $(L, M)$ a representing pair when $L$ is a representing group.

If $G$ is finite then it must be generated by a finite set of subgroups $A_{1}, A_{2}, \ldots, A_{n}$. Let $\left(L_{i}, M\left(H_{i}\right)\right)$ be a representing pair for $A_{i}, \quad i=1,2, \ldots, n$. Then these can be used to form a representing pair for $G$ when $G$ is a regular product.

We use the same constructions as before. There is an epimorphism from $L_{i}$ to $A_{i}$ with kernel $M\left(A_{i}\right)$ by hypothesis. Let $L=\prod_{i=1}^{n} L_{i}$ and $\sigma$ be the natural epimorphism from $L$ onto $A$ induced by these epimorphisms. Put $J=H_{1} \cap\left[L[L]\right.$, where $H_{I}$ is the group in $L$ which maps onto $H$ under $\sigma$, and let $N=\prod_{\substack{i, j=1 \\ i \neq j}}^{n}\left[M\left(A_{i}\right), L_{j}\right]^{L}$. Then Jo=H is normal in $L$.

THEOREM 3.1. Suppose $G$ is finite and a regular product of its subgroups $A_{1}, A_{2}, \ldots, A_{n} \cdot$ Let $\left(L_{i}, M\left(A_{i}\right)\right)$ be a fixed representing pair for $A_{i}$. Then $(L \tau, M \tau)$ is a representing pair for $G$ where 
$\tau: L \rightarrow L / N[J, L]$ and $M=\left(\prod_{i=1}^{n} M\left(A_{i}\right)\right) . J$ in $L$. The groups $L, N$ and $J$ are as defined above.

Proof. (i) Apply Lemma 2.1 by putting $I=\{1,2, \ldots, n\}, B_{i}=L_{i}$ and $C_{i}=M\left(A_{i}\right)$. Then $L=B, C=M, N=D$ and $J=K$. We have

$$
\begin{aligned}
G & \simeq B / C \quad \text { from Lemma } 2.1 \\
& \simeq \frac{B / D[K, B]}{C / D[K, B]} \quad \text { since } D[K, B] \leq C \text { from (2.3) (i). }
\end{aligned}
$$

Thus $G=\frac{L / N[J, L]}{M / N[J, L]}=L \tau / M \tau$ by definition of $\tau$.

Now $J$ is a subgroup of $L^{\prime}$ immediately from its definition, and $M\left(A_{i}\right)$ is a subgroup of $L_{i}^{\prime}$ by hypothesis, so that $M$ is a subgroup of $L^{\prime}$. That is $(M \tau)^{\prime}$ is a subgroup of $(L \tau)^{\prime}$.

The kernel of $\tau$ contains $[J, L]$ as a subgroup which means that $J \tau$ is central in $L \tau$. Also $\left[M\left(A_{i}\right), L_{j}\right], i \neq j$, is a subgroup of $N$ which is in ker $\tau$. Thus $M\left(A_{i}\right) \tau$ commutes with $L_{j} \tau$ for $i \neq j$. If $i=j$ then $M\left(A_{i}\right)$ commutes with $L_{i}$ by hypothesis. Hence $M \tau$, as a product of $J \tau$ and the $M\left(A_{i}\right) \tau$, is central in $L \tau$.

The pair $(L \tau, M \tau)$ will be a representing pair if $M_{\tau}$ is isomorphic to $M(G)$.

(ii) Theorem 2.5 gives that

$$
M \cap L^{\prime} /[M, L] \simeq\left(\prod_{i=1}^{n} \times\left(M\left(A_{i}\right) \cap L_{i}^{\prime}\right) /\left[M\left(A_{i}\right), L_{i}\right]\right) \times H /[H, A] .
$$

But $M\left(A_{i}\right) \leq L_{i}^{\prime} \cap Z\left(L_{i}\right)$ so that $M\left(A_{i}\right) \cap L_{i}^{\prime}=M\left(A_{i}\right)$ and $\left[M\left(A_{i}\right), L_{i}\right]=E$. Hence

$$
\begin{aligned}
M \sim L^{\prime} /[M, L] & \simeq\left(\prod_{i=1}^{n} \times\left(A_{i}\right)\right\} \times H /[H, A] \\
& \simeq M(G)
\end{aligned}
$$

by Theorem 2.6 .

Looking at the quotient $M L^{\prime} /[M, L]$, we have from (2.3) (ii), that 


$$
\begin{aligned}
{[M, L] } & =\left(\prod_{\substack{i, j=1 \\
i \neq j}}^{n}\left[M\left(A_{i}\right), L_{j}\right]\right][J, L] \\
& =N[J, L] \\
& =\operatorname{ker\tau } .
\end{aligned}
$$

by definition

Thus $M \tau=M \cap L^{\prime} /[M, L] \simeq M(G)$.

Theorem 3.1 has already been established by Wiegold in [10] for the case where $G=A_{1} \times A_{2}$.

\section{An application of Theorem 2.6}

Blackburn has calculated the multiplicator of a wreath product $A$ wr $B$ for arbitrary groups $A$ and $B$ in [1]. I can obtain a more general result for a verbal wreath product, $A \mathrm{wr}_{V} B$, using techniques similar to those of Section 2. However, when $A$ is abelian, $A \mathrm{wr}_{V} B$ is isomorphic to a regular product of $A$ and $B$ and $M\left(A w r_{V} B\right)$ can be found directly using Theorem 2.6.

Let $A_{b}$ be an isomorphic copy of $A$ for each $b \in B$ and denote by $a_{b}$ the element of $A_{b}$ mapped to $a \in A$. The $V$-verbal product $D=\prod_{b \in B}^{V} A_{b}$ corresponding to a set of words $V$ is defined to be the quotient $C / C_{V}$ where $C=\prod_{b \in B}^{*} A_{b}$ and $C_{V}=V(C) \cap\left[A_{b}^{C}\right]$ (see Moran [5]). The mapping $a_{b} \mapsto a_{b b^{\prime}}$ for all $a \in A, b \in B$ and fixed $b^{\prime} \in B$, induces automorphisms of both $C$ and $D$.

DEFINITION 4.1. The free wreath product of $A$ by $B, A \mathrm{wr}_{*} B$, is the splitting extension of $C$ by $B$ under the above action of $B$ on $C$. Similarly the V-verbal wreath product $A \mathrm{wr}_{V} B$, is the splitting extension of $D$ by $B$ under the action of $B$ on $D$.

Actually $A \mathrm{wr}_{V} B=A \mathrm{wr}_{*} B$ when $V$ is the empty word.

The verbal wreath product is generated by $A_{1}$ and $B$ since 
$a_{b}=b^{-1} a_{1} b$ for all $a \in A, b \in B$. It is therefore a quotient of $A * B$ by some group $H$. Now both $\left[A_{b}^{C}\right]$ and $V(C)$ are invariant under the action of $B$ on $C$ by definition of the cartesian and since verbal subgroups are characteristic. That is $C_{V}=V(C) \cap\left[A_{b}^{C}\right]$ is normal in $A \mathrm{wr}_{*} B$. It follows from Definition 4.1 that $A \mathrm{wr}_{V} B$ is isomorphic to $A \mathrm{wr}_{*} B / C_{V}$. But it is well known that the natural epimorphism from $A * B$ onto $A$ wr $B$ induced by the identity on $B$ and the isomorphism $A \stackrel{\sim}{\rightarrow} A_{1}$, is also an isomorphism (Hall and Hartley [4]). Hence $H=C_{V} \phi^{-1}$ and,

(4.2). $A \mathrm{wr}_{V} B$ is isomorphic to $A \star B / C_{V} \phi^{-1}$.

By (1.8) $A \mathrm{wr}_{V} B$ will be a regular product of $A$ and $B$ if $C_{V} \phi^{-1}$ is contained in the cartesian $[A, B]$, or equivalently, if $C_{V}$ is contained in $[A, B] \phi$. Firstly

$$
\begin{aligned}
{[A, B] \phi^{-1} } & =\operatorname{sgp}\left([a, b] \phi^{-1} \mid a \in A, b \in B\right) \\
& =\operatorname{sgp}\left(a_{1}^{-1} a_{b} \mid a \in A, b \in B\right) \\
& =\operatorname{sgp}\left(a_{b}^{-1} a_{b}, \mid a \in A ; b, b^{\prime} \in B\right) .
\end{aligned}
$$

Also, by (1.2), $\left[A_{b}^{C}\right]$ is the normal closure in $C$ of the commutators $\left[a, a_{b}^{\prime}\right], a, a^{\prime} \in A ; b, b^{\prime} \in B$. But

$$
\begin{aligned}
{\left[a_{b}, a_{b^{\prime}}^{\prime}\right] } & =a_{b}^{-1} a_{b^{\prime}}^{\prime-1} a_{b} a_{b^{\prime}}^{\prime} \\
& =\left(a_{b}^{-1} a_{b^{\prime}}\right) a_{b^{\prime}}^{-1} a_{b^{\prime}}^{\prime-1} a_{b} a_{b}^{\prime}\left(a_{b}^{\prime-1} a_{b^{\prime}}^{\prime}\right) \\
& =\left(a_{b}^{-1} a_{b^{\prime}}\right)\left[a_{b^{\prime}}, a_{b^{\prime}}^{\prime}\right] a_{b^{\prime}}^{\prime-1} a_{b^{\prime}}^{-1} a_{b} a_{b}^{\prime}\left(a_{b}^{\prime-1} a_{b^{\prime}}\right) \\
& =\left(a_{b}^{-1} a_{b^{\prime}}\right)\left[a, a^{\prime}\right]_{b^{\prime}}\left(\left(a a^{\prime}\right)_{b^{\prime}}\right)^{-1}\left(a a^{\prime}\right)_{b}\left(a_{b}^{\prime-1} a_{b^{\prime}}^{\prime}\right) \\
& =\left(a_{b}^{-1} a_{b^{\prime}}\right)\left(\left(a a^{\prime}\right)_{b^{\prime}}\right)^{-1}\left(a a^{\prime}\right)_{b}\left(a_{b}^{\prime-1} a_{b^{\prime}}^{\prime}\right)
\end{aligned}
$$


for all $a, a^{\prime} \in A$ if and only if $A$ is abelian. Thus $[A C]$ is contained in $[A, B] \phi$ and $A \mathrm{wr}_{V} B$ is indeed a regular product of $A$ and $B$.

THEOREM 4.3. If $A$ is abelian, the multiplicator of $A \mathrm{wr}_{V} B$ is isomorphic to $M(A) \times M(B) \times C_{V} /\left[C_{V}, C\right]\left[C_{V}, B\right]$ where $C_{V}=V(C) \cap\left[A_{b}^{C}\right]$, $C=\prod_{b \in B}^{*} A_{b}$ and the quotient is evaluated in $A \mathrm{wr}_{*} B$.

Proof. From Theorem 2.6 the multiplicator of $A * B / C_{V} \phi^{-1}$ is $M(A) \times M(B) \times C_{V^{\phi^{-1}}} /\left[C_{V^{\phi^{-1}}}, A * B\right]$. Also

$$
\begin{aligned}
C_{V} \phi^{-1} /\left[C_{V} \phi^{-1}, A * B\right] & \simeq C_{V} /\left[C_{V}, A \text { wr } * B\right] \\
& =C_{V} /\left[C_{V}, C . B\right] \text { since } A \text { wr }_{*} B \\
& =C_{V} /\left[C_{V}, C\right]\left[C_{V}, B\right] .
\end{aligned}
$$

The ordinary wreath product $A$ wr $B$ is obtained from $A$ wr $_{V} B$ when $V(C)=C^{\prime}$ and $D$ becomes the direct product $\prod_{b \in B}^{x} A_{b}$.

COROLLARY 4.4. If $A$ is abelian, $M(A$ wr $B)$ is isomorphic to $M(A) \times M(B) \times\left(\prod_{b<b^{\prime}}^{\times} A_{b} \otimes A_{b^{\prime}}\right) / N$ where "<" is any fixed ordering of the elements of $B$ and $N$ is the subgroup generated by the elements

$$
\left.\begin{array}{l}
\left(a_{b} \otimes a_{b^{\prime}}^{\prime}\right)^{-1}\left(a_{b b^{\prime \prime}} \otimes a_{b^{\prime} b^{\prime \prime}}\right) \quad \text { when } b b^{\prime \prime}<b^{\prime} b^{\prime \prime} \\
\left(a_{b} \otimes a_{b^{\prime}}^{\prime}\right)^{-1}\left(a_{b^{\prime} b^{\prime \prime}} \otimes a_{b b^{\prime \prime}}\right)^{-1} \text { when } b^{\prime} b^{\prime \prime}<b b^{\prime \prime}
\end{array}\right\}
$$

for all $a, a^{\prime} \in A ; b, b^{\prime}, b^{\prime \prime} \in B$.

Proof. When $V(C)=C^{\prime}$, denote $C_{V}$ by $c_{x}$ and let $\alpha$ be the canonical homomorphism from $A \mathrm{wr}_{*} B$ onto $A \mathrm{wr}_{*} B /\left[C_{x}, C\right] ;\left(\left[C_{x}, C\right]\right.$ is 
normal since $C$ is normal). The quotient $C_{V} /\left[C_{V}, C\right]\left[C_{V}, B\right]$ is isomorphic to $C_{x} \alpha /\left[C_{x}, B\right] \alpha$.

$$
\text { Now } C_{x}=C^{\prime} \cap\left[\begin{array}{l}
C \\
A_{b}
\end{array}\right]=\left[\begin{array}{c}
C \\
b
\end{array}\right] \text {, so that } C \alpha \text { is the second nilpotent }
$$

product, $\prod^{(2)}{ }_{A}$, of the $A_{b}$ (see [6]). We have the following elementary facts:

(i) $\left[A_{b}^{C}\right] \alpha$ is central in $C \alpha$, by construction of $\alpha$;

(ii) $\left[A_{b}^{C}\right] \alpha=\prod_{b, b}\left[A_{b}, A_{b},\right] \alpha$ by definition of the cartesian and (i);

(iii) $\left[A_{b}, A_{b^{\prime}}\right] \alpha=\left[A_{b^{\prime}}, A_{b}\right] \alpha$;

(iv) $\left[A_{b_{1}}, A_{b_{1}^{\prime}}\right] \alpha \cap \operatorname{sgp}\left(\left[A_{b_{2}}, A_{b_{2}^{\prime}}\right] \alpha \mid b_{2}, b_{2}^{\prime} \in B\right.$

$$
\left.\left(b_{2}, b_{2}^{\prime}\right) \neq\left(b_{1}, b_{1}^{\prime}\right),\left(b_{1}^{\prime}, b_{1}\right)\right)
$$

is trivial by Definition 1.1 and the associativity of nilpotent products [2];

(v) $\left[A_{b}, A_{b},\right] \simeq A_{b} \otimes A_{b}$, by the remark following Theorem 2.6 .

Therefore $\left[A_{b}^{C}\right] \alpha$ is isomorphic to $\prod_{b<b^{\prime}}^{\times} A_{b} \otimes A_{b^{\prime}}$.

Finally,

$$
\begin{aligned}
{\left[C_{x}, B\right] \alpha } & =\operatorname{sgp}\left(c^{-1} c^{\prime \prime} \mid c \in\left[A_{b}^{C}\right], b^{\prime \prime} \in B\right) \alpha \\
& =\operatorname{sgp}\left(\left(\left[a_{b}, a_{b}^{\prime},\right]^{-1}\left[a_{b}, a_{b}^{\prime}\right]^{b^{\prime \prime}}\right) \alpha \mid a \in A ; b, b^{\prime}, b^{\prime \prime} \in B\right) \\
& =\operatorname{sgp}\left(\left(\left[a_{b}, a_{b^{\prime}}^{\prime}\right]^{-1}\left[a_{b b^{\prime \prime}}, a_{b^{\prime}}^{\prime} b^{\prime \prime}\right]\right) \alpha \mid a \in A ; b, b^{\prime}, b^{\prime \prime} \in B\right) .
\end{aligned}
$$


The quotient $C_{x} \alpha /\left[C_{x}, B\right] \alpha$ is clearly isomorphic to $\left(\prod_{b<b^{\prime}}^{\times} A_{b} \otimes A_{b^{\prime}}\right) / N$.

\section{References}

[1] Norman Blackburn, "Some homology groups of wreathe products", Illinois J. Math. 16 (1972), 116-129.

[2] O.N. Golovin, "Nilpotent products of groups", Amer. Math: Soc. Transt. (2) 2 (1956), 89-115.

[3] Marshall Hall, Jr, The theory of groups (The Macmillan Company, New York, 1959).

[4] P. Hall and B. Hartley, "The stability group of a series of subgroups", Proc. London Math. Soc. (3) 16 (1966), 1-39.

[5] S. Moran, "Associative operations on groups. I", Proc. London Math. Soc. (3) 6 (1956), 381-596.

[6] S. Moran, "Associative operations on groups. II", Proc. London Math. Soc. (3) 8 (1958), 548-568.

[7] J. Schur, "Über die Darstellung der endlichen Gruppen durch gebrochene lineare Substitutionen", J. reine angew. Math. 127 (1904), 20-50.

[8] J. Schur, "Untersuchungen über die Darstellungen der endlichen Gruppen durch gebrochene lineare Substitutionen", J. reine angew. Math. 132 (1907), 85-137.

[9] James Wiegold, "Nilpotent products of groups with amalgamations", Pub 2. Math. Debrecen 6 (1959), 131-168.

[10] James Wiegold, "The multiplicator of a direct product", Quart. J. Math. Oxford (2) 22 (1971), 103-105.

Department of Pure Mathematics, School of General Studies, Australian National University, Canberra, ACT. 